\title{
Herramienta didáctica para integrar las TIC en la enseñanza de las ciencias
}

\author{
César Augusto Gutiérrez ${ }^{1}$
}

Recibido: 20-02-2018

Aceptado: 24-04-2018

\section{RESUMEN}

En los retos actuales que se plantean para la educación, se debe reconocer el papel que desempeñan la lectura, la escritura y las TIC en el proceso de enseñanza-aprendizaje de las ciencias naturales. Este artículo describe un trabajo que se desarrolla en torno a la comprensión del movimiento parabólico simple, utilizando la interdisciplinariedad entre física y lenguaje con el objetivo de relacionar los métodos científicos y las competencias comunicativas al quehacer diario en la escuela. Siguiendo una investigación cuasi-experimental con enfoque cuantitativo, se aplicó una encuesta a los estudiantes de grado décimo de una Institución Educativa en la ciudad de Santiago de Cali - Colombia, donde se logró identificar como principal necesidad, dinamizar el proceso de enseñanzaaprendizaje de la física, involucrando actividades mediadas por TIC que fortalezcan las competencias científicas. El proyecto se planteó como una respuesta proactiva y exploratoria a la necesidad de mejorar e innovar los métodos de enseñanza-aprendizaje y dar respuesta a los retos que son planteados por los estudiantes en la actualidad. Como resultados tras la aplicación de la herramienta diseñada, se logró evidenciar la mejoría en el interés por el tema y las actividades propuestas en la clase, además de un fortalecimiento en las pruebas evaluativas aplicadas periódicamente.

Palabras clave: Estrategia didáctica; ciencias naturales; TIC; Objeto virtual de aprendizaje 


\title{
Didactic tool to integrate ICT in the teaching of science
}

\begin{abstract}
In the current challenges that arise for education, the role played by reading, writing and ICT in the teaching-learning process of the natural sciences must be recognized. This article describes a work that develops around the understanding of the simple parabolic movement, using the interdisciplinarity between physics and language with the objective of relating scientific methods and communicative competences to the daily tasks in school. Following a quasi-experimental research with a quantitative approach, a survey was applied to tenth grade students of an Educational Institution of Santiago de Cali - Colombia, where it was possible to identify as the main need, boost the teaching-learning process of the physical, involving ICT-mediated activities that strengthen scientific competencies. The project was proposed as a proactive and exploratory response to the need to improve and innovate teachinglearning methods and respond to the challenges that are posed by current students. As results after the application of the designed tool, it was possible to demonstrate the improvement in interest in the subject and the activities proposed in the class, as well as a strengthening in the evaluation tests applied periodically.
\end{abstract}

Keywords: Didactic strategy; Natural sciences; Virtual learning object ICT

\section{Ferramenta didática para integrar as TIC no ensino de ciências}

\section{RESUMO}

Nos desafios atuais que surgem para a educação, o papel desempenhado pela leitura, escrita e TIC no processo ensino-aprendizagem das ciências naturais deve ser reconhecido. Este artigo descreve um trabalho que se desenvolve em torno da compreensão do movimento parabólico simples, usando a interdisciplinaridade entre física e linguagem com o 
objetivo de relacionar métodos científicos e competências comunicativas às tarefas diárias na escola. Após uma pesquisa quase- experimental, com abordagem quantitativa, uma pesquisa foi aplicado aos alunos do segundo ano de grau escola en Cali - Colômbia, que foi identificado como uma necessidade premente, agilizar o processo de ensino e aprendizagem física, envolvendo atividades mediadas por TIC que fortaleçam as competências científicas. O projeto foi proposto como uma resposta proativa e exploratória à necessidade de melhorar e inovar os métodos de ensino-aprendizagem e responder aos desafios que os alunos atuais representam. Como resultados após a aplicação da ferramenta projetada, foi possível demonstrar a melhoria do interesse no assunto e as atividades propostas na classe, bem como um fortalecimento nos testes de avaliação aplicados periodicamente.

Palavras-chave: Estratégia didática; Ciências Naturais; TIC; Objeto de aprendizagem virtual.

\section{Introducción}

En la educación media, los estudiantes acceden a diferentes tipos de textos que requieren niveles avanzados de competencias comunicativas (Barrantes, Beltrán, \& Pérez, 2016), así pues, los docentes deben proponer didácticas que vayan orientadas a facilitar la comprensión de los conceptos del área a través de actividades que faciliten la adquisición del conocimiento e integren diferentes saberes del currículo, Galagovsky (2001), confirma que una de las debilidades de los estudiantes, es que no tienen las habilidades necesarias para la comprensión y producción de textos en las áreas de ciencias como la física.

Por otro lado, Ruiz (2007), menciona que el modelo tradicional de enseñanza por transmisión - recepción, se encuentra aún arraigado en el contexto educativo actual, sin embargo, la juventud de la actualidad tiene características diferentes como el manejo innato de las herramientas TIC, el espíritu investigador, inquieto, la creatividad, entre otras (Aparicio \& Ostos, 2018); lo que exige generar nuevas propuestas didácticas pertinentes, para incentivar el proceso de enseñanza-aprendizaje de las ciencias naturales y que permitan una mejor apropiación de los conceptos como: 1) el modelo por investigación que reconoce una estructura interna en donde se identifica claramente problemas de orden científico y se 
pretende que éstos sean un soporte fundamental para la secuenciación de los contenidos a ser enseñados, además se identifica claramente su postura constructivista y la aplicación de problemas para la enseñanza de las ciencias, 2) el modelo por descubrimiento, donde se pueden distinguir dos matices, la primera denominada modelo por descubrimiento guiado, donde le brindamos al estudiante los elementos requeridos para que él encuentre la respuesta a los problemas planteados o a las situaciones expuestas y le orientamos el camino que debe recorrer para dicha solución; o autónomo cuando es el mismo estudiante quien integra la nueva información y llega a construir conclusiones originales, 3) modelo de recepción significativa donde se hace una relación directa de la lógica interna de la ciencia con la lógica del aprendizaje del educando, es decir se piensa que la manera cómo se construye la ciencia es compatible con el proceso deaprendizaje desarrollado por el educando, generando la idea de compatibilidad entre el conocimiento científico y el cotidiano y 4) el cambio conceptual que reconoce una estructura cognitiva en el educando, al valorar sus pre-saberes y se introduce un nuevo proceso desde la enseñanza de las ciencias mediante el conflicto cognitivo (Aparicio \& Ostos, 2018).

En la investigación sobre pedagogía y didáctica se encontraron propuestas con estrategias interesantes como el Aprendizaje por Indagación, que, en cuanto a su evaluación del aprendizaje tiene dos propósitos, uno formativo y otro sumativo. El primero, o evaluación para el aprendizaje, tiene la función de apoyar al estudiante donde intervienen procesos de búsqueda e interpretación de evidencia y que permite al docente observar dónde está el estudiante, para dónde debe ir y cuál es la mejor forma de llegar al conocimiento, y el segundo llamado evaluación del aprendizaje, determina lo que el estudiante ha aprendido hasta un determinado momento a través de pruebas. (Harlen, 2003). Por otro lado, la Pedagogía de la Comprensión planteada por Perkins (2008), tiende a desarrollar competencias o habilidades del pensamiento como lo hace la enseñanza por indagación, y es que ambas, tienen su foco centrado en la capacidad del ser humano de ser autónomo en su aprendizaje y en la capacidad innata que tiene para aprender, ambas ofrecen al maestro formas diferentes de enseñar; en ellas hay un cambio de rol del docente tanto como del estudiante. Sumado a estas, se integra la propuesta de los profesores Punya Mishra y Mattew Koehler (2006), de la Universidad Estatal de Michigan sobre el modelo TPACK acrónimo de "Technological Pedagogical and Content Knowledge", el cual menciona la integración de las TIC en el proceso de enseñanza-aprendizaje a través de entornos, 
objetos y herramientas virtuales, como los simuladores, aplicaciones y programas con fines educativos.

Proponer actividades enmarcadas en una didáctica que permita a los estudiantes llevar procesos de aprendizaje más activos a través del desarrollo de competencias comunicativas y científicas, tomando como herramientas recursos tecnológicos, es necesario para dar un giro en las estructuras tradicionales de las clases magistrales.

En los cursos de secundaria se motiva al estudiante a que continúe leyendo y escribiendo, sin embargo, se hace necesario brindarles insumos académicos para hacerlo cada vez mejor, de tal modo que fortalezcan estas competencias como lo plantea González y Laguado (2007), ya que se evidencian niveles bajos de desempeño en las competencias básicas de lenguaje y en los procesos que llevan a la comprensión de las temáticas planteadas y que en muchos casos persisten en las diferentes asignaturas a lo largo de su paso por la educación básica (Tahull, 2016).

Lo anterior se evidencia en muchas de las actividades que desarrollan los estudiantes en su proceso formativo en la secundaria y se deja notar en el informe nacional "Saber $3^{\circ}, 5^{\circ}$ y $9^{\circ}$ Resultados nacionales 20092014" (ICFES, 2016), sobre los resultados de las pruebas saber.

Esta problemática se extiende a los grados $10^{\circ}$ y $11^{\circ}$, ya que, los estudiantes deben asumir lecturas de textos que les exigen niveles y procesos de comprensión más complejos, necesarios para la solución de problemas relacionados con situaciones científicas para diseñar y planear estrategias que les permitan aplicar modelos matemáticos y llegar a soluciones verificables.

El problema de la interpretación y de la comprensión se hace evidente en la falta de habilidades en las competencias básicas que evalúa el Ministerio de Educación Nacional (MEN, 2006), para el área de lenguaje y que influyen de forma negativa en el desarrollo de destrezas para el área de ciencias, por ejemplo, en los indicadores de desempeño planteados para física, donde se deben desarrollar competencias en las ciencias exactas.

La prueba SABER 11 evalúa el desarrollo de competencias básicas en los ciclos $3^{\circ}, 5^{\circ}$ y $9^{\circ}$ y están enfocadas en valorar las siguientes competencias en las áreas de lenguaje, matemáticas y ciencias 
Tabla 1. Competencias evaluadas por el ICFES.

\begin{tabular}{|c|c|c|}
\hline Lenguaje & Matemáticas & Ciencias Naturales \\
\hline $\begin{array}{c}\text { Competencia comunicativa a } \\
\text { través de dos procesos: } \\
\text { Lectura Escritura }\end{array}$ & $\begin{array}{c}\text { Razonamiento y argumentación } \\
\text { Comunicación, representación y } \\
\text { modelación } \\
\text { Planteamiento y resolución de } \\
\text { problemas }\end{array}$ & $\begin{array}{c}\text { Uso comprensivo del conocimiento } \\
\text { científico Explicación de } \\
\text { fenómenos Indagación }\end{array}$ \\
\hline
\end{tabular}

Fuente. Información suministrada por el equipo de evaluación de la calidad de la Secretaría de desarrollo pedagógico de la Secretaría de Educación Municipal de Santiago Cali. (Rosas, 2015).

En el año 2015 los estudiantes de grado once, presentaban promedios bajos en el área de matemáticas, destacando la competencia de razonamiento y argumentación en un nivel básico y la de planteamiento y resolución de problemas en un nivel insuficiente. Igualmente, la Subsecretaría de Desarrollo Pedagógico del municipio indicó que la mayor parte del problema radicaba en las prácticas de aula utilizadas por los docentes (Rosas, 2015).

En el siguiente cuadro se muestran los resultados globales de la prueba saber 11 del año 2015 para la Institución investigada.

Tabla 2. Cantidad de estudiantes evaluados año 2016.

\begin{tabular}{|c|c|c|c|c|c|}
\hline Código DANE & Nivel del Reporte & Matriculados & Registrados & Presentes & Publicados \\
\hline & $\begin{array}{c}\text { Colombia (8979 } \\
\text { establecimientos) }\end{array}$ & 426747 & 466071 & 461287 & 456351 \\
\hline $\begin{array}{c}\text { Cali (283 } \\
\text { Establecimientos) }\end{array}$ & 20486 & 16405 & 16193 & 15947 \\
\hline $\begin{array}{c}\text { Institución } \\
\text { Educativa Santo } \\
\text { Tomás }\end{array}$ & 131 & 131 & 128 & 128 \\
\hline
\end{tabular}

Fuente. Datos suministrados por el equipo pedagógico de la SEM de Santiago de Cali. (Rosas, 2015).

Tabla 3. Resultados globales de la prueba saber 11-año 2015

\begin{tabular}{|c|c|c|c|c|c|c|c|c|}
\hline Código DANE & Nivel de Reporte & $\begin{array}{l}\text { Lectura critica } \\
\text { Promedio } \\
\text { (Desviación) }\end{array}$ & $\begin{array}{l}\text { Matemá ticas } \\
\text { Promedio } \\
\text { (Desviación) }\end{array}$ & $\begin{array}{c}\text { Sociales y } \\
\text { ciudadanas } \\
\text { Promedio } \\
\text { (Desviación) }\end{array}$ & $\begin{array}{c}\text { Ciencias } \\
\text { naturales } \\
\text { Promedio } \\
\text { (Desviación) }\end{array}$ & $\begin{array}{c}\text { Inglés } \\
\text { Promedio } \\
\text { (Desviación) }\end{array}$ & $\begin{array}{l}\text { Razonamiento } \\
\text { cuantitativo } \\
\text { (Desviación) }\end{array}$ & $\begin{array}{l}\text { Competencias } \\
\text { ciudadanas } \\
\text { (Desviación) }\end{array}$ \\
\hline \multirow{6}{*}{176001040079} & \multirow{2}{*}{$\begin{array}{c}\text { Colombia } \\
\text { (8979 } \\
\text { establecimientos) }\end{array}$} & 50.7 & 51.5 & 51 & 51.3 & 51.3 & 52.6 & 50.4 \\
\hline & & $(7,5)$ & $(9.3)$ & $(9.1)$ & (7.7) & (7.8) & (10.6) & (8.3) \\
\hline & \multirow{2}{*}{$\begin{array}{c}\text { Cali (283 } \\
\text { establecimientos) }\end{array}$} & 50.6 & 49.7 & 50.1 & 50.2 & 50.1 & 50.8 & 49.9 \\
\hline & & (7.6) & (9) & (9) & (7.8) & (8) & (10.3) & (8.1) \\
\hline & \multirow{2}{*}{$\begin{array}{l}\text { INSTITUCIÓN } \\
\text { EDUCATIVA } \\
\text { SANTO TOMÁS }\end{array}$} & 53.3 & 51.6 & 53 & 51.4 & 50.4 & 52.7 & 52.5 \\
\hline & & $(7.3)$ & $(8.2)$ & (8.9) & (7.8) & (9) & (9.7) & (7.8) \\
\hline
\end{tabular}

Fuente. Datos suministrados por el equipo pedagógico de la SEM de Santiago de Cali. (Rosas, 2015). 
Los resultados de la Institución Educativa son similares a los promedios municipal y nacional, pero ello no se puede considerar como un logro definitivo teniendo en cuenta que a nivel nacional los resultados obtenidos no alcanzan el nivel que se requiere para posicionarse como un país líder en el desarrollo de educación de calidad.

En el siguiente gráfico se muestra un leve progreso de los estudiantes de la institución educativa investigada, durante los tres últimos años, en los resultados de las pruebas específicas de lectura crítica y ciencias naturales.

A pesar del leve progreso, es necesario seguir elevando los niveles de desempeño en las competencias de lectura y escritura con miras a que las demás asignaturas se beneficien y sigan contribuyendo al progreso en los promedios de las evaluaciones que realiza el ICFES (de Almeida, Santos y Porto, 2016).

Gráfico 1. Resultados prueba saber 11 2014-2016.

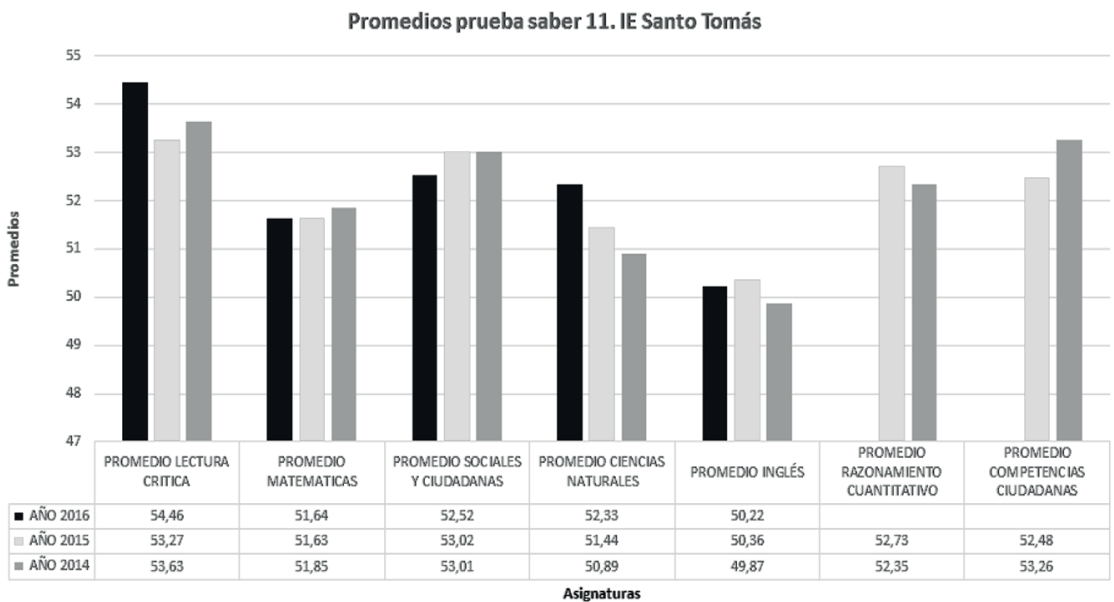

Fuente. Rosas, 2015.

En cuanto a las pruebas PISA (OCDE, 2006), los resultados de la evaluación de la competencia lectora, se dividieron en tres rubros según el tipo de proceso que los reactivos pedían. Así se obtuvo una puntuación en recuperación de información, otra en interpretación de textos, y una más en reflexión y evaluación. Además, se ofrece una evaluación global de la competencia lectora en una escala que tiene una media de 500 puntos y en la que la gran mayoría de los estudiantes se ubican entre los 
300 y los 700 puntos. Integrando los tres tipos de procesos evaluados, se obtuvo la siguiente escala general en el que se distinguen 6 niveles de desempeño:

Tabla 4. Niveles de desempeño en comprensión lectora, pruebas PISA.

\begin{tabular}{|c|c|c|}
\hline NIVEL & PUNTAJE & CARACTERISTICAS \\
\hline $\begin{array}{l}\text { Nivel } 5 \text {, el } \\
\text { más alto }\end{array}$ & con 625 puntos o más & $\begin{array}{l}\text { En él se ubican los estudiantes que pueden manejar } \\
\text { información difícil de encontrar en textos con los que no } \\
\text { están familiarizados. } \\
\text { Son estudiantes que muestran una comprensión detallada } \\
\text { de dichos textos y pueden inferir qué información del texto } \\
\text { es relevante para responder al reactivo. Pueden recurrir } \\
\text { a conocimiento especializado, evaluar críticamente y } \\
\text { establecer hipótesis. }\end{array}$ \\
\hline Nivel 4 & de 553 a 625 puntos & $\begin{array}{c}\text { Alumnos que pueden responder a reactivos difíciles, como } \\
\text { los que piden ubicar información escondida o interpretar } \\
\text { significados a partir de sutilezas del lenguaje. Pueden } \\
\text { evaluar críticamente un texto. }\end{array}$ \\
\hline Nivel 3 & de 481 a 552 puntos & $\begin{array}{l}\text { Son capaces de trabajar con reactivos de complejidad } \\
\text { moderada. Ubican fragmentos múltiples de información, } \\
\text { vinculan distintas partes de un texto y relacionan dicho } \\
\text { texto con conocimientos familiares o cotidianos. }\end{array}$ \\
\hline Nivel 2 & de 408 a 480 puntos & $\begin{array}{l}\text { Los alumnos responden reactivos básicos como los que } \\
\text { piden ubicar información directa, realizar inferencias } \\
\text { sencillas, identificar lo que significa una parte bien definida } \\
\text { de un texto y utilizar algunos conocimientos externos para } \\
\text { comprenderla. }\end{array}$ \\
\hline Nivel 1 & de 335 a 407 puntos & $\begin{array}{l}\text { En ese nivel están los alumnos que sólo pueden ubicar } \\
\text { un fragmento de información, identificar el tema principal } \\
\text { de un texto y establecer una conexión sencilla con el } \\
\text { conocimiento cotidiano. }\end{array}$ \\
\hline $\begin{array}{l}\text { Por debajo } \\
\text { del nivel } 1\end{array}$ & menos de 335 puntos & $\begin{array}{c}\text { Están los alumnos que pueden leer, en el sentido técnico } \\
\text { de la palabra, pero que tienen importantes dificultades } \\
\text { para utilizar la lectura como una herramienta que les } \\
\text { permita ampliar sus conocimientos y destrezas en } \\
\text { diferentes áreas. }\end{array}$ \\
\hline
\end{tabular}

Fuente. Elaboración propia.

Con respecto al informe de las pruebas PISA para el año 2015 (El País, 2016), Colombia se encuentra en las competencias en Ciencias en 416 , en comprensión lectora en 425 y en competencias en matemáticas 390, lo que indica que se encuentra muy alejado para alcanzar una medida razonable.

De igual forma, De Zubiría (2016), considera que el nivel mínimo que debe tener un ciudadano para poder interactuar adecuadamente en el contexto actual es el 2. Pero en lectura, el 43 por ciento de los estudiantes colombianos no lo alcanza y en matemáticas el 66 por ciento tampoco lo logra. 
Al mismo tiempo, en lectura solo 9 de cada mil jóvenes llegan al nivel de crítica y solo el 7 por ciento alcanza el nivel de lectura profunda.

Los esquemas de aprendizaje requieren que los estudiantes vayan desarrollando gradualmente sus niveles de interpretación, que en muchas ocasiones no se llegan a dar en los momentos esperados, ya que los factores externos impiden que estos se construyan; por otro lado, los modelos y los enfoques pedagógicos y didácticos que se imparten en el aula, en muchas ocasiones no alcanzan a indagar más allá, sobre la forma en cómo los estudiantes aprenden y sobre qué estrategias se está dando este aprendizaje.

Afrontar un problema de la física requiere de un esquema organizacional donde además de interpretar gráficamente la situación planteada, se deben identificar e interpretar las variables que intervienen con sus respectivos valores numéricos, las incógnitas del problema y las ecuaciones que se deben considerar.

Aunque el contexto se presenta sencillo en estas líneas, la realidad muestra que cuando en el aula se presenta una situación - problema propio de la física, (de baja o mediana complejidad) los estudiantes tienen dificultades para abordarla.

Se pueden enumerar diversos factores que influyen, como son: los bajos niveles de interpretación y argumentación textual, pocas habilidades comunicativas, deficientes bases matemáticas, mal uso de la calculadora, alta desatención o desinterés por el conocimiento que se está impartiendo, una metodología poco didáctica u otros factores asociados a problemáticas familiares o sociales que frenan los procesos académicos de los estudiantes.

Presentar a los estudiantes actividades donde se hagan transversales varias estrategias de las asignaturas del currículo, es una propuesta pertinente que puede resultar provechosa para un mejoramiento de la didáctica de la física en el salón de clases.

La Física, como una de las ramas de la ciencia naturales, necesita de la experimentación y la indagación para poder llegar a conclusiones generales que fundamenten las leyes de la naturaleza, pero si un problema o una teoría no son bien interpretados pueden generar confusiones o malos cálculos, esto conlleva a sentimientos de fracaso y rechazo hacia 
la asignatura, se puede así estar perdiendo la oportunidad de que los estudiantes desarrollen competencias científicas, que le permitan trabajar con los errores y aprender de la situación.

Al respecto de las TIC, la OCDE (2006), menciona que las pueden ayudar a mejorar el aprendizaje, si se articulan a estrategias como el trabajo por proyectos, basados en la investigación escolar, facilitando el aprendizaje cooperativo, metodologías de evaluación del aprendizaje, aprovechando en definitiva las TIC para acercar las ciencias a los estudiantes, con simuladores, laboratorios de experimentación virtual, entre otros recursos educativos digitales. (Bedoya, 2016). En los últimos años, desde el Ministerio de Educación Nacional las instituciones educativas, han sido incluidas en programas de formación para docentes en el uso pedagógico de las TIC, dentro de los más reconocidos están, el programaTit@, diplomados de Computadores Para Educar y la Fundación Telefónica, entre otros.

El Ministerio TIC en apoyo al proceso de inclusión de la educación en las nuevas formas de comunicación, ha impulsado a los docentes para que integren estas herramientas en el aula de clases, dotando de tabletas o computadores y formando a los docentes en un uso adecuado por medio de diplomados. Tras un año de haber sido beneficiadas con tecnología, las escuelas y colegios públicos empiezan a presentar mejores resultados sobre la tasa de deserción, la tasa de repitencia, el desempeño en Pruebas de Conocimiento Saber y las tasas de acceso a la educación superior. Las TIC siguen impactando positivamente la educación colombiana. (MINTIC, 2016) Estas iniciativas además de ser formativas han dotado de herramientas tecnológicas, como computadoras, tabletas, tableros digitales y proyectores (Konieczny, 2015).

Consultando a algunos maestros de diferentes instituciones educativas se ha podido concluir que en la mayoría de casos estos dispositivos están siendo subutilizados, o no se utilizan por temor al deterioro, y no se les da el uso potencial para el que fueron creados, de la misma manera un alto porcentaje de docentes no aprovechan las capacitaciones, ni tampoco las instituciones apoyan adecuadamente a los maestros en estos procesos.

El auge de la tecnología ha traído consigo una mar de posibilidades para que los docentes se apropien de los recursos necesarios para cambiar las prácticas en el aula e implementen nuevas formas de organizar y presentar sus clases y plantear las actividades a los estudiantes (Pérez, 2013). 
El proyecto propuso presentar a estudiantes de grado décimo, el movimiento parabólico desde estrategias de indagación, comprensión e interpretación textual para el análisis de situaciones problema, antes de llegar al procedimiento matemático. Es así como a través del ejercicio de las competencias de lenguaje y de ciencias naturales e integrando a estas los recursos y equipos computacionales, se pretende mejorar los procesos didácticos para la solución de problemas de física para promover un esquema donde prevalezca un fortalecimiento de las competencias comunicativas y científicas antes que la adquisición plana de una temática.

Grisolia (2008), en su artículo "La Interdisciplinaridad en la Enseñanza de las Ciencias", publicado en la revista Ciencia y Educación en marzo, toma referentes de varios autores (Visser (2002), Piaget, citado por Ricci (2003), Falla (1999), Lértora (2002) y Reyes(2001)) sobre la diferencia entre interdisciplinariedad, multidisciplinariedad, y transdisciplinariedad de los cuales deduce que los tres términos hacen referencia a la cooperación entre dos o más disciplinas para la resolución de un problema, además dice, que la transdisciplinariedad se distingue de las otras dos, en que en ella se construye un nuevo campo de saber que combina varias disciplinas tomando identidad propia, la interdisciplinariedad es la condición didáctica que permite cumplir el principio de la sistematización de la enseñanza y asegurar el reflejo consecuente de las relaciones objetivas vigentes en la naturaleza y la sociedad, mediante el contenido de las diferentes asignaturas que integran el currículo de la escuela actual. (Fiallo, 1997), por tanto, se hace pues necesaria la interdisciplinariedad en la escuela, donde de manera integrada áreas como la física, el lenguaje y la informática se unan para cumplir una labor educativa, evidenciando los aportes de cada una de ellas en la construcción del conocimiento, mediante actividades de carácter metodológico.

Según el Diccionario de la educación y métodos de enseñanza, Carderera (2009) se conoce el Trivium y el Quadrivium como programas pioneros de una enseñanza integrada. Se le llamó así a la forma como se dividió la enseñanza en la escuela durante la edad media, el Trívium comprendía la gramática, la dialéctica y la retórica, mientras que el Quadrivium incluía la aritmética, la geometría, la astronomía y la música. A estas siete disciplinas se les llamó las artes liberales porque, según el sabio inglés Salisbury, quien las dominaba era conocedor de los caminos para llegar a la sabiduría. Se cita a Bacon quien plantea la necesidad de unificar el saber (Torres, 1994). Se desarrolló un ambiente virtual 
educativo que integra el uso de la física y el lenguaje como herramientas útiles, prácticas, reflexivas y motivadoras para la resolución significativa de problemas en pro de la enseñanza y aprendizaje de ambas asignaturas, para ello se plantea la propuesta usando una de las temáticas de la física del grado décimo, el movimiento parabólico, pretendiendo que sea la base para temáticas posteriores propias de esta disciplina de las ciencias naturales.

Se espera que docentes de matemáticas, física y lenguaje conozcan la presente propuesta, la implementen con los ajustes que ellos consideren pertinentes, se sientan motivados a cambiar las prácticas pedagógicas en el aula y se mejoren los procesos de enseñanza y el aprendizaje de estas asignaturas.

Por todo lo anterior, el objetivo principal del proyecto fue proponer una estrategia didáctica que promueva la comprensión del movimiento parabólico en la asignatura de física, integrada con las TIC y apoyada en los procesos de lectoescritura, en la educación media técnica.

\section{Metodología}

El desarrollo del proyecto se dividió en varias etapas que permitieron en cada una de ellas recopilar información necesaria para documentar el proceso de investigación, definir el tema a tratar y plantear la hipótesis sobre la problemática con la documentación bibliográfica donde se consultaron y se referenciaron varios autores que habían trabajado sobre el tema, esto permitió confrontar con la realidad del aula y definir las técnicas e instrumentos para plantear la propuesta. Los estudiantes que participaron en la implementación del proyecto cursan grado décimo cuyas edades oscilan entre los 15 y 18 años, algunos son vecinos del nororiente de la ciudad o vienen de barrios o lugares cercanos a la ciudad de Cali.

Los datos recopilados de la población participante sirvieron como insumos para diseñar la propuesta didáctica y los instrumentos de aprendizaje para que sea implementada durante el año lectivo. Se recurrió a varias fuentes primarias como libros, monografías, tesis y publicaciones oficiales, documentales, videocintas y fuentes secundarias como resúmenes de referencias primarias. (Hernández, Collado y Lucio, 2006). Todo el proceso de recolección de información se hizo durante 
gran parte del año lectivo 2016 por parte del docente del área de física, mediante los resultados académicos evidenció las competencias con mayor índice de reprobación y la aplicación de dos encuestas donde se buscaba caracterizar el impacto de las TIC en su quehacer diario, además se realizó un protocolo de observación que en conjunto arrojó resultados más concretos a la motivación e interés por cada clase.

La encuesta que se utilizó como instrumento de recolección de información fue fundamental en este proyecto, con esta se buscaba conocer la perspectiva de los estudiantes sobre las estrategias didácticas aplicadas actualmente en el proceso de enseñanza aprendizaje, lo cual permitió a los investigadores validar la necesidad de plantear la propuesta. Se realizó un cuestionario, de ocho preguntas, diseñados en Google Drive y se dispusieron en los grupos de Edmodo ya existentes, para su diligenciamiento. La muestra fue aleatoria y libre, se conformó automáticamente con un total de 29 participantes con respecto a la población de 125 estudiantes, lo que refiere un 25\% aproximadamente.

Después de identificar las competencias a trabajar, se utilizaron las propias del área ciencias naturales presentadas por el MEN, así como las competencias digitales.

\section{Estándares básicos de competencias con enfoque interdisciplinar (MEN, 2004)}

- Me aproximo al conocimiento como científico-a natural Utilizo modelos biológicos, físicos y químicos para explicar la transformación y conservación de la energía.

-Manejo conocimientos propios de las ciencias naturales Identifico aplicaciones de diferentes modelos Biológicos, químicos y físicos en procesos industriales y en el desarrollo tecnológico; analizo críticamente las implicaciones de sus usos.

- Medios de comunicación y otros sistemas simbólicos Retomo críticamente los lenguajes no verbales para desarrollar procesos comunicativos intencionados.

- Comprensión e interpretación textual Comprendo e interpreto textos con actitud crítica y capacidad argumentativa. 
-Producción textual

Produzco textos argumentativos que evidencian mi conocimiento de la lengua y el control sobre el uso que hago de ella en contextos comunicativos orales y escritos.

\section{Competencias $e$ indicadores de desempeño Competencias de ciencias naturales y educación ambiental}

- Uso comprensivo del conocimiento científico Identifico variables que influyen en la experimentación con lanza-proyectiles.

- Explicación de fenómenos Establezco relaciones entre los datos de la experimentación con datos teóricos del movimiento parabólico.

- Indagación

Expreso de diversas formas el proceso de experimentación con fenómenos parabólicos.

\section{Competencias específicas}

La elaboración del OVA tiene en cuenta la integración de las competencias específicas:

Tabla 5. Enfoque por competencias del OVA.

\begin{tabular}{|c|c|c|}
\hline \multicolumn{3}{|c|}{ COMPETENCIAS ESPECÍFICAS } \\
\hline SABER SER & SABER CONOCER & SABER HACER \\
\hline $\begin{array}{l}\text { Trabaja en cooperación para } \\
\text { conseguir un objetivo. } \\
\text { Desempeñas roles activos en el } \\
\text { trabajo en colaboración. } \\
\text { Tiene autonomía en la } \\
\text { resolución de problemas. } \\
\text { Busca alternativas para } \\
\text { alcanzar un objetivo. }\end{array}$ & $\begin{array}{c}\text { Construye conocimiento } \\
\text { científico a través de la } \\
\text { indagación. } \\
\text { Observa y elabora instrumentos } \\
\text { para la recolección de datos. } \\
\text { Aplica métodos de observación } \\
\text { científica. } \\
\text { Usa la expresión escrita con } \\
\text { propiedad para elaborar } \\
\text { textos de carácter científico e } \\
\text { investigativo. }\end{array}$ & $\begin{array}{l}\text { Escribe informes de carácter } \\
\text { científico. } \\
\text { Desarrolla laboratorios } \\
\text { siguiendo un proceso científico. } \\
\text { Explora el contexto y analiza la } \\
\text { problemática que se le plantea. } \\
\text { Busca alternativas de solución a } \\
\text { los problemas planteados. }\end{array}$ \\
\hline
\end{tabular}

Fuente. Elaboración propia. 


\section{Objetivos de aprendizaje}

Los objetivos de aprendizaje enmarcan los propósitos principales de la implementación del proyecto en el aula, estos corresponden a una integración de las ciencias naturales y las TIC.

- Promover en los estudiantes el uso de herramientas virtuales que permitan su autoaprendizaje y guíen el proceso de indagación científica sobre el movimiento parabólico.

- Inducir la comprensión del movimiento parabólico a través de una didáctica que involucra laboratorios físicos y virtuales.

- Integrar una didáctica que involucre las áreas de lenguaje y física para la comprensión del movimiento parabólico, tomando como partida actividades de indagación y observación científica de los fenómenos físicos.

- Promover habilidades científicas y de comprensión en los estudiantes por medio de un objeto virtual de aprendizaje y del estudio del movimiento parabólico.

\section{Presentación del producto final}

El objeto virtual de aprendizaje está diseñando con el programa eXeLearning, y responde a una didáctica de la Pedagogía de la Comprensión y al aprendizaje por indagación sobre el movimiento parabólico. El objeto está dirigido para estudiantes de educación media en Colombia y responde a la búsqueda de un aprendizaje significativo que parte del contexto colombiano.

Las actividades están elaboradas para incentivar las competencias en las áreas de ciencias naturales y lenguaje; fueron organizadas en una secuencia que avanza en torno a la indagación (Figura 1), y la observación científica, a la par, incluyen la lectura, escritura y argumentación. Las herramientas que predominan en el OVA son Power Point, Powtoon, Genially, Educaplay, diversos Idivices de eXeLearning, Google Drive, entre otras. 
Figura 2. Estructura del OVA



Fuente. Elaboración propia.

Las actividades y contenidos tratan subtemas que tienen que ver con el movimiento parabólico como las variables, constantes y ángulos en las ecuaciones y la forma en que se resuelven, sumando a esto, incluye laboratorios que tienen como objetivo principal experimentar con los fenómenos del movimiento parabólico, a través de simuladores que complementan la práctica del docente en el aula (Viejo, Cabezas \& Martínez, 2013).

Figura 3. Página principal del OVA

\begin{tabular}{|c|c|}
\hline $\begin{array}{l}\text { EXPLORANDO EL } \\
\text { MOVIMIENTO PARABÓLICO }\end{array}$ & Z Observa el siguiente vídeo \\
\hline LA FÍSICA EN LA VIDA COTIDIANA & \multirow{12}{*}{$\begin{array}{l}\text { En la escuela muchas veces nos hablan de cosas que tal vez aprendemos sin encontrarle } \\
\text { alguna relación con las situaciones que vivimos o vemos diariamente, pero no nos detenemos } \\
\text { quizá a pensar que todo lo que sucede tiene alguna explicación científica o una razón de } \\
\text { ser. } \\
\text { La física por ejemplo, muchas veces se nos convierte en una de esas asignaturas por la cual } \\
\text { debemos responder para que nuestro nivel académico sea el mejor, pero isí has notado que } \\
\text { está en toda parte?. El solo hecho de hablar o escuchar es un proceso donde interviene el } \\
\text { medio por donde viaja el sonido, si no hubieses ese medio, que es el aire, no podríamos } \\
\text { comunicarnos. Cuando vas en una patineta y deseas bajarte debes hacerlo en la dirección } \\
\text { del movimiento para que no vayas a sufrir una caída, esto responde a la ley de la inercia. } \\
\text { Cada } 24 \text { horas vivimos un nuevo día y cada } 365 \text { días un nuevo año, esto se debe a que la } \\
\text { Tierra rota y se traslada simultáneamente respondiendo a las características del } \\
\text { movimiento circular... podríamos continuar hablando de las múltiples aplicaciones que tiene } \\
\text { la física en todos los campos de nuestra vida, de todas formas puedes explorar muchos } \\
\text { campos de aplicación de ésta fascinante ciencia, por ahora te invito a que le explores este } \\
\text { objeto virtual de aprendizaje y realices todas las actividades que te planteamos. } \\
\text { jBIENVENIDOs! }\end{array}$} \\
\hline $\begin{array}{l}\text { Estándares con enfoque } \\
\text { interdisciplinar }\end{array}$ & \\
\hline $\begin{array}{l}\text { Competencias e indicadores de } \\
\text { desempeño }\end{array}$ & \\
\hline $\begin{array}{l}\text { 1. Activa tus saberes: la fisica en los } \\
\text { goles }\end{array}$ & \\
\hline $\begin{array}{l}\text { 2. Laboratorio físico experimenta con } \\
\text { el entorno }\end{array}$ & \\
\hline 3. Interpreta y afianza tus habilidades & \\
\hline $\begin{array}{l}\text { 4. Laboratorio Virtual: juega con } \\
\text { simuladores }\end{array}$ & \\
\hline 5. Simuladores virtuales & \\
\hline $\begin{array}{l}\text { 6. Autoevaluación: Mide tu } \\
\text { aprendizaje }\end{array}$ & \\
\hline 7. Pasatiempos & \\
\hline 8. Rompecabezas & \\
\hline Referencias de imágenes & \\
\hline
\end{tabular}

Fuente. Elaboración propia. 


\section{Resultados y discusión}

Dentro de los resultados obtenidos durante la investigación, se pueden destacar dos apartes principalmente, el primero es la percepción que tienen actualmente los estudiantes sobre las didácticas utilizadas para la enseñanza de las ciencias naturales y la importancia de la integración de las TIC en estos procesos, fruto del diligenciamiento de una encuesta por 29 estudiantes de grado décimo de la Institución Educativa.

\section{Encuesta}

Tabla 6. Encuesta-P 1

1. ¿Cuál de los siguientes recursos tecnológicos es el que más usa usted como ayuda para realizar susconsultas y tareas?

\begin{tabular}{|c|c|}
\hline Teléfono celular & $44.8 \%$ \\
\hline Tableta & $0 \%$ \\
\hline Computador & $24.1 \%$ \\
\hline Todos los anteriores & $31 \%$ \\
\hline
\end{tabular}

Fuente. Elaboración propia.

Resultado: El 45\% de los estudiantes encuestados utiliza principalmente el celular como instrumento para realizar sus consultas, el $24 \%$ utiliza principalmente el computador, mientras que el $31 \%$ tienen la posibilidad de usar el teléfono, la tableta, el computador.

Conclusión: La totalidad de los estudiantes tienen acceso a la tecnología para realizar sus consultas y tareas.

Tabla 7. Encuesta-P 2

2. ¿Cuál de los siguientes usos es el que usted más le da a su celular?

\begin{tabular}{|c|c|}
\hline No tiene celular & $27.6 \%$ \\
\hline Para chatear & $24.1 \%$ \\
\hline Para hacer consultas por internet & $37.9 \%$ \\
\hline Para escuchar música & $7 \%$ \\
\hline Para hacer llamadas & $3 \%$ \\
\hline
\end{tabular}

Fuente. Elaboración propia. 
Resultado: El 27\% no tiene celular, el $24 \%$ lo usa para chatear, el $37 \%$ para hacer consultas por internet, el $7 \%$ para escuchar música y el $3 \%$ para llamar.

Conclusión: Un poco más de la cuarta parte de los estudiantes no poseen celular pero posiblemente acceden desde sus casas para hacer sus consultas y tareas. Aquellos que lo tienen además de usarlo con fines académicos también le dan algún uso recreativo o dispositivo de comunicación con otras personas.

Tabla 8. Encuesta-P 3

\begin{tabular}{|c|c|}
\hline \multicolumn{2}{|c|}{ 3. ¿Cuándo uso el celular en el colegio? } \\
\hline Es restringido por todos los docentes durante las clases & $10.3 \%$ \\
\hline Es restringido por algunos docentes & $27.6 \%$ \\
\hline Solo se puede usar si es con fines educativos & $62.1 \%$ \\
\hline Está prohibido así sea con fines educativos & $0 \%$ \\
\hline
\end{tabular}

Fuente. Elaboración propia.

Resultado: El 10\% dice que todos los docentes restringen el uso del celular, el $8 \%$ afirman que algunos docentes lo restringen y el $62 \%$ confirma que los docentes permiten el uso sólo si es con fines académicos.

Conclusión: Aunque el celular no es restringido en su totalidad, los estudiantes sienten que no hay libertad total para su uso permanente durante la jornada escolar.

Tabla 9. Encuesta-P 4

\begin{tabular}{|c|c|}
\hline \multicolumn{2}{|c|}{$\begin{array}{l}\text { 4. ¿En cuáles de las siguientes asignaturas el profesor permite el uso del celular para el } \\
\text { desarrollo de la clase? (puede señalar varias opciones) }\end{array}$} \\
\hline Física & $93.3 \%$ \\
\hline Química & $46.7 \%$ \\
\hline Matemáticas & $36.7 \%$ \\
\hline Español & $36.7 \%$ \\
\hline Inglés & $73.3 \%$ \\
\hline Sociales & $0 \%$ \\
\hline Ciencias Económicas & $3.3 \%$ \\
\hline Artística & $30 \%$ \\
\hline
\end{tabular}

Fuente. Elaboración propia. 
Resultado: El 93\% dicen usar el celular para el desarrollo de la clase de física, $73 \%$ en inglés, $47 \%$ en química, $40 \%$ en español, $37 \%$ en matemáticas, $30 \%$ en artística, $3 \%$ en ciencias económicas y no es permitido en sociales

Conclusión: en algunas asignaturas el nivel de uso del celular como herramienta para el desarrollo de las clases es baja o casi nula, no se solicitó información por la asignatura tecnología e informática pues su uso ya controlado era del $100 \%$.

Tabla 10. Encuesta-P 5

5. ¿Qué tan importante es para usted que los docentes utilicen recursos tecnológicos para el desarrollo de las clases?

\begin{tabular}{|c|c|}
\hline Muy importante & $56.7 \%$ \\
\hline Importante & $43.3 \%$ \\
\hline Poco importante & $0 \%$ \\
\hline Nada importante & $0 \%$ \\
\hline
\end{tabular}

Fuente. Elaboración propia.

Resultado: El 57\% consideran muy importante el uso de la tecnología para el desarrollo de las clases, el $43 \%$ lo consideran importante.

Conclusión: Para los estudiantes el uso de las TIC en el desarrollo de las clases es necesario. Posiblemente se sienten motivados cuando se propone su uso.

Tabla 11. Encuesta-P 6

6. Cuando los docentes asignan actividades donde hay que usar la internet usted..

\begin{tabular}{|c|c|}
\hline Realiza con agrado la actividad y siente que aprende más & $80 \%$ \\
\hline Cumple con la actividad pero no es tan importante & $7 \%$ \\
\hline Se le dificulta porque no le gusta la tecnología & $0 \%$ \\
\hline Se le dificulta porque no tiene internet en su casa & $13.3 \%$ \\
\hline
\end{tabular}

Fuente. Elaboración propia.

Resultado: El $80 \%$ de los estudiantes gustan del uso de la internet en las actividades académicas y sienten que se aprende más, el $13 \%$ se le dificulta por no tener acceso permanente en su casa mientras que el $7 \%$ aunque cumplen no lo ven como algo importante. Ninguno manifiesta apatía por el uso de la internet. 
Conclusión: El uso de la internet ayuda a que los estudiantes realicen sus consultas académicas y profundicen en las temáticas abordadas.

Tabla 12. Encuesta-P 7

7. ¿Qué tanto le gustaría que las clases de física involucrarán en sus actividades lecturas y actividades que se deben resolver en un dispositivo móvil?

\begin{tabular}{|c|c|}
\hline Mucho & $76.7 \%$ \\
\hline Le es indiferente & $13.3 \%$ \\
\hline No le gustaría & $10 \%$ \\
\hline
\end{tabular}

Fuente. Elaboración propia.

Resultado: El 77\% de los estudiantes les gustaría involucrar en las clases de física actividades integradoras de lectura y el uso del celular, al 13\% le es indiferente y al $10 \%$ no le gustaría.

Conclusión: La mayoría de los estudiantes desean cambios en la didáctica de la física, que sea integradora con el lenguaje y el uso del dispositivo móvil.

Tabla 13. Encuesta-P 8

\begin{tabular}{|c|c|}
\hline $\begin{array}{c}\text { 8.¿Por qué cree usted que algunos docentes no usan los equipos tecnológicos de la institución } \\
\text { para el desarrollo de las clases? }\end{array}$ \\
\hline Los docentes no están capacitados & $16.7 \%$ \\
\hline Los docentes le huyen a la tecnología & $0 \%$ \\
\hline La conexión a internet en el colegio no es buena & $70 \%$ \\
\hline Por temor a que se dañen los equipos & $13.3 \%$ \\
\hline
\end{tabular}

Fuente. Elaboración propia.

Resultado: El $70 \%$ de los estudiantes tienen la percepción que los docentes no usan los recursos tecnológicos disponibles por parte de la institución educativa debido a la pésima conectividad existente, El 17\% creen que los docentes no están capacitados en el uso de la tecnología y el $13 \%$ consideran que no lo hacen por temor al daño o deterioro de los equipos.

Conclusión: Se debe mejorar la conectividad en la institución educativa.

El segundo aparte refleja la percepción del grupo investigador y docente mediante un protocolo de observación realizado durante el proceso de implementación del proyecto, el cual tuvo una duración aproximada de 5 semanas para un total de 20 horas de clase. 


\section{Protocolo de observación en la implementación}

Tabla 14. Protocolo de observación de implementación.

\begin{tabular}{|l|l|l|}
\hline \multicolumn{3}{|c|}{ PROTOCOLO DE OBSERVACIÓN } \\
\hline Proyecto: & Observador: & \\
\hline Lugar: & Escena: & \\
\hline Hora inicio: & \\
\hline Hora final: & \\
\hline Descripción & \\
\hline & \\
\hline Interpretativo & \\
\hline & \\
\hline Temático & \\
\hline
\end{tabular}

Fuente. Elaboración propia.

Del anterior protocolo aplicado se pudieron evidenciar los siguientes hallazgos:

- Se fortaleció el trabajo colaborativo por parte de los estudiantes en el desarrollo de las actividades propuestas, cada integrante realizaba aportes significativos del tema.

- Se evidenció un alto interés por las temáticas, donde no solo se limitan a realizar lo propuesto, sino que realizaban investigación para generar nuevos conocimientos que les permitan mejorar la entrega de la actividad en desarrollo.

- El uso de actividades interactivas como videoquices, crucigramas, simuladores, entre otros, fomentan el espíritu competitivo de los estudiantes, donde se esfuerzan por lograr el mejor resultado. 
- La disciplina de los estudiantes mejoró, al aumentar su interés por la clase, participaban de forma activa, lo que conllevaba a tener un menor porcentaje de estudiantes realizando actividades inoficiosas o perturbando el buen ambiente de trabajo.

- Cuando no había una buena conectividad y no se podía acceder a todas las actividades de manera óptima, se evidenciaba la perdida de interés.

- En algunas oportunidades, la falta de infraestructura o espacios con dispositivos tecnológicos retrasaba el proceso.

\section{Conclusiones}

Después de lograr una implementación de la estrategia pedagógica propuesta se pudo concluir que:

- Esta propuesta buscó que los estudiantes que ingresan a la educación media técnica, de la institución educativa Santo Tomás, tengan la posibilidad, de acceder al conocimiento científico a través de actividades que requieran el uso de competencias de lectura y escritura mediadas por el uso de las tecnologías de la información y la comunicación.

- Se diseñó un objeto virtual de aprendizaje para la clase de física donde se plantea una didáctica para abordar el conocimiento del movimiento parabólico, usando estrategias de lectura, escritura y herramientas TIC. EI OVA contiene actividades integradoras donde prevalece la pedagogía de la indagación como estrategia de aprendizaje; su estructura puede ser llevada a otras temáticas que se plantean no solo desde las ciencias naturales, sino desde cualquier otra área del conocimiento.

- La población estudiantil participante que motivó la presentación de la propuesta son jóvenes que oscilan entre 
los 15 y los 18 años, para ellos el uso de la tecnología se hace indispensable en cada actividad de la cotidianidad, los métodos de enseñanza no pueden ser los mismos con los que aprendieron sus padres y maestros, por eso se hace necesaria la implementación de nuevas alternativas que marchen paralelamente a las opciones tecnológicas de la época (Langer, 2016).

- La clase de física junto con la de química, en la educación media, son la representación del área de ciencias naturales, pero a medida que se presentan las temáticas se va desligando como elemento constitutivo y se transforma, en otra rama de las matemáticas, dejando a un lado el desarrollo de competencias científicas como son: el uso comprensivo del conocimiento científico, la explicación de fenómenos y la indagación.

- Las nuevas propuestas didácticas siempre serán una oportunidad de mejorar el proceso de enseñanzaaprendizaje, sobre todo si se integran las TIC, ya que los estudiantes del presente manejan las herramientas de forma innata, y es labor fundamental del educador, que lo hagan de una forma responsable y productiva.

\section{Recomendaciones}

- En el área de las ciencias naturales plantear propuestas didácticas que incluyan razonamientos y además promuevan, en los estudiantes, el desarrollo de las competencias propias, disminuyendo el protagonismo a la cuantificación de los resultados.

- Implementar durante el siguiente año lectivo nuevamente la propuesta en las clases de física de los estudiantes que ingresan a la educación media técnica de la institución educativa Santo Tomás, subsanando las dificultades encontradas en la primera oportunidad. 
- Hacer seguimiento y presentar los resultados de la implementación del OVA en una matriz que enfatice los aciertos y desaciertos con sus respectivas recomendaciones para futuras experiencias.

\section{Referencias bibliográficas}

Aparicio, O.Y., \& Ostos, O.L. (2018). Las TIC como herramientas cognitivas para la investigación. Revista Interamericana de Investigación, Educación y Pedagogía, RIIEP, 11(1). https://orcid.org/0000-0003-3535-6288. https://orcid.org/0000-0002-6477-9872

Barrantes, H. A., Beltrán, J. E. P., \& Pérez, F. A. R. (2016). Perfil del estudiante de pregrado de la Facultad de Estudios a Distancia de la Universidad Militar Nueva Granada. Revista Interamericana de Investigación, Educación y Pedagogía, RIIEP, 9(2). DOI: https://doi.org/10.22490/25391887.1948

Bedoya, R. F. (2016) Entornos digitales y políticas educativas dilemas y certezas. EI impacto de las TIC en la educación. El caso de computadores para educar. UNESCO. Recuperado de: https://goo.gl/SSMwLt

Carderera. M. (2009). Diccionario de educación y métodos de enseñanza. Madrid: Imprenta de A. Vicente.

de Almeida, R. R., Santos, M. F., \& Porto, J. C. (2016). Lectura de textos ficcionales y el enfoque escolar de literatura: contribuciones para una Pedagogía de la Elección. Revista Interamericana de Educación, Pedagogía y Estudios Culturales, 9(1), 35-51. DOI: https://doi.org/10.22490/25391887.1925

Konieczny, P. (2015). Lorenzo García Aretio: bases, mediaciones y futuro de la educación a distancia en la sociedad digital. Revista Interamericana de Investigación, Educación y Pedagogía, RIIEP, 8(1). DOI: https://doi.org/10.15332/s1657-

107X.2015.0001.08

Pérez, T. H. P. (2013). Aproximaciones al estado de la cuestión de la investigación en educación y derechos humanos. Revista Interamericana de Investigación, Educación y Pedagogía, RIIEP, 6(1). DOI: https://doi.org/10.15332/s1657-107X.2013.0001.05

Tahull, J. (2016). Modernidad, educación y género. El proyecto inacabado. Revista Interamericana de Investigación, Educación y Pedagogía, RIIEP, 9(2), 159-178. DOI: https://doi.org/10.22490/25391887.1947

De Zubiría, J. (2016) Resultados de las pruebas PISA: no hay mucho que celebrar. Razón Pública, 11 (2) Recuperado de: https://www.razonpublica.com/index.php/economia-ysociedad/9923-resultados-de-las-pruebas-pisa-no-hay-mucho-que-celebrar.html 
El País (2016). Resultados del informe PISA (online), Madrid. Obtenido de: http://elpais. com/elpais/2016/12/05/media/1480958752_164797.html

Fiallo, J. (1997). La interdisciplinariedad. Reto para la calidad de un currículo. Revista Desafío Escolar, 2 (23) mayo-julio.

Galagovsky, y Adúriz-Bravo, (2001). Modelos y analogías en la enseñanza de las ciencias naturales: El concepto de modelo didáctico analógico. 19(2), 231-242. Recuperado de https://ddd.uab.cat/record/1527

González \& Laguado. (2007) Documentos. MEN. Obtenido de: https://goo.gl/Ef3r2S

Grisolia, M. (2008). La interdisciplinariedad en la enseñanza de las ciencias.

Recuperado de. https://goo.gl/u1tupm

Harlen, W. (2003). Evaluación y Educación en Ciencias Basada en la Indagación: Aspectos de la Política y la Práctica. Recuérado de https://goo.gl/jvrmDX

Hernández, R, Collado C. y Lucio P. (2006) Metodología de la investigación. Recuperado de: https://goo.gl/xSXE41

Langer, E. (2016). La construcción de confianza para el estudio de prácticas de resistencia en la escolarización de jóvenes en contextos de pobreza urbana. Revista Interamericana de Investigación, Educación y Pedagogía, RIIEP, 9(2). DOI: https://doi. org/10.22490/25391887.1945

MEN (2004). Estándares básicos de competencias en ciencias naturales y ciencias sociales. Ministerio de Educación Nacional de Colombia.

MEN (2006). Estándares básicos de competencias en lenguaje, matemáticas y ciencias ciudadanas. Guía sobre lo que los estudiantes deben saber y saber hacer con lo que aprenden. Ministerio de Educación Nacional de Colombia.

MINTIC (2016). Las TIC siguen impactando positivamente la educación colombiana. Sala de Prensa. Recuperado de: https://goo.gl/VU42C2

Mishra, P., y Koehler, M. J. (2006). Technological pedagogical content knowledge: A framework for teacher knowledge. Teachers College Record, 108(6), 1017-1054. DOI: https://doi.org/10.1111/j.1467-9620.2006.00684.x

OCDE, P. (2006). El programa PISA de la OCDE qué es y para qué sirve. Santillana.

OCDE, PISA. (2009). Marco de la evaluación: Conocimientos y habilidades en ciencias, Matemáticas y Lectura. Paris: OCDE.

Perkins, D. (2008). La escuela inteligente. Editorial Gedisa S.A. España. 
Ruíz, F. J. (2007). Modelos didácticos para la enseñanza de las ciencias naturales. Revista Latinoamericana de Estudios Educativos 3(2).

Rosas, J. (2015), Asistencia técnica a la Institución Santo Tomás. Gestión de la evaluación para la calidad del servicio educativo. Secretaria de educación municipal de Santiago de Cali. Cali. Colombia.

Viejo, C. M., Cabezas, I. L., \& Martínez, M. D. J. I. (2013). Las redes de académicas en la docencia universitaria. Revista Interamericana de Investigación, Educación y Pedagogía, RIIEP, 6(2). DOI: https://doi.org/10.15332/s1657-107X.2013.0002.03 